\title{
Average properties of combinatorial problems and thermodynamics of spin models on graphs
}

\author{
Alessandro Vezzani, Davide Cassi and Raffaella Burioni \\ Dipartimento di Fisica, Istituto Nazionale di Fisica della Materia (INFM), Università di Parma, parco Area delle \\ Scienze 7A 43100 Parma Italy \\ vezzaniefis.unipr.it
}

\begin{abstract}
The study of thermodynamic properties of classical spin models on infinite graphs naturally leads to consider the new combinatorial problems of random-walks and percolation on the average. Indeed, spin models with $O(n)$ continuous symmetry present spontaneous magnetization only on transient on the average graphs, while models with discrete symmetry (Ising and Potts) are spontaneously magnetized on graphs exhibiting percolation on the average. In this paper we define the combinatorial problems on the average, showing that they give rise to classifications of graph topology which are different from the ones obtained in usual (local) random-walks and percolation. Furthermore, we illustrate the theorem proving the correspondence between Potts model and average percolation.
\end{abstract}

Keywords: statistical mechanics, graphs, random-walks, percolation

\section{Introduction}

The interplay between physics and geometry is one of the most interesting and fascinating problem of theoretical modern physics. On lattices many important results have been achieved in this direction, indeed, it is well known that the euclidean dimension determines many aspects of the behaviour of physical systems. In particular, for classical spin models, the existence itself of spontaneous magnetization depends on lattice dimensionality. In this context classical rigorous results are the Mermin-Wagner (MW) and Fröhlich-Simon-Spencer (FSS) theorems which prove that classical spin models with $O(n)$ continuous symmetry are spontaneously magnetized only on a lattice with dimension $d \geq 3$. On the other hand Peierls-Griffi ths argument (PG) proves that discrete symmetry spin models (Ising and Potts) present spontaneous magnetization when $d \geq 2$.

The study of physical properties of inhomogeneous systems such has glasses, polymers and biological systems naturally leads to consider statistical models defi ned on generic networks, i.e. on graphs $(\mathrm{H}$; B; CDS). ¿From this point of view an extension to graphs of the results (MW; FSS; PG) is of great interest. Unfortunately, the geometry of inhomogeneous networks is not described by a simple and well defi ned parameter, as it is the euclidean dimension for lattices. Therefore, the characterization of the topology requires the introduction of new criteria. Important results $(\mathrm{Ca} ; \mathrm{BCV} 2 ; \mathrm{V})$ show that simple combinatorial 
problems give rise to graph classifi cations which turn out to be very useful to understand the behaviour of physical models. In particular, continuous symmetry spin models are related to random-walks, and discrete symmetry spin models to percolation.

In statistical physics we are mainly interested in the thermodynamic properties of the systems defi ned by extensive functions, such as the average magnetization, the free energy, the magnetic susceptibility, the heat capacity. These quantities depend on the values of the spins through the average over all the sites of the graph. Therefore, graph classifi cations, which correctly describe the thermodynamic behaviour, must be defi ned introducing combinatorial problems on the average.

In $(\mathrm{Ca}$; $\mathrm{BCV} 2)$ it is put into evidence that classical spin models with continuous $O(n)$ symmetry are spontaneously magnetized only on graphs where random-walks are transient on the average. Unfortunately, argument (BCV2) is not rigorous, however a physical insight into the problem well justifi es the result. Furthermore, random-walks on the average are related to other important physical problems such as harmonic oscillations (AO), the Gaussian (HHW; BBC) and the spherical (CF) models. On the other hand, a recent theorem $(\mathrm{V})$ proves that discrete symmetry models (Ising and Potts) are spontaneously magnetized on graphs presenting percolation on the average.

Random-walks and percolation on the average are defi ned by averaging, over all sites of the graph, the probability of returning to the starting point in $t$ steps and the probability of belonging to a cluster of size larger than $l$, respectively. On homogeneous structures, such as lattices, these probabilities are equal for all the vertices, therefore, average and usual (local) combinatorial problems coincide. However, on inhomogeneous structures they give rise to different classifi cations of graph topology, as it will be shown in details in the paper.

Averages on infi nite graphs are introduced by means of the thermodynamic limit. An important mathematical open question regards the existence of the limit itself. ¿From a physical point of view we assume that it is always well defi ned, since only in this case the systems exhibit a proper thermodynamical behaviour. Nevertheless, it would be very interesting to get the general conditions for its existence.

Random-walks and percolation on the average lead to different classifi cations of graph topology. Indeed, it is well known that lattices presenting percolation (i.e. lattices with dimension $d \geq 2$ ) are transient only if $d \geq 3$. Recently (S) it has been proved that the "anomalous" behaviour of presenting average percolation in a recurrent on the average structure is typical not only of two dimensional lattices but also of "low dimensional networks", such as the Sierpinski carpet (Fig. 1). Therefore, a general result clarifying the relation between the two classifi cations is still lacking.

In this paper we defi ne the combinatorial problems of random-walks (BCV1) and percolation (V) on the average showing the differences with the usual local defi nitions. We will always underline the main mathematical open questions, especially concerning the existence of the thermodynamic limit. Furthermore, we will illustrate the theorem showing the relation between percolation on the average and the Potts model. This result is inspired by a mathematical paper (Hä) where it is proven an analogous relation between (local) percolation and the existence of a unique Gibbs measure for the Potts model, which is a typical local property not related to the thermodynamics of the system.

\subsection{Definitions and notations}

Let us introduce some defi nitions and notations that will be useful in the following (H; B; CDS).

Definition 1 A graph $X$ is a countable set $V_{X}$ of vertices (or sites) $i$ connected pairwise by a set $E_{X}$ of unoriented edges (or links) $(i, j)=(j, i)$. Two connected vertices are called nearest neighbours. We 


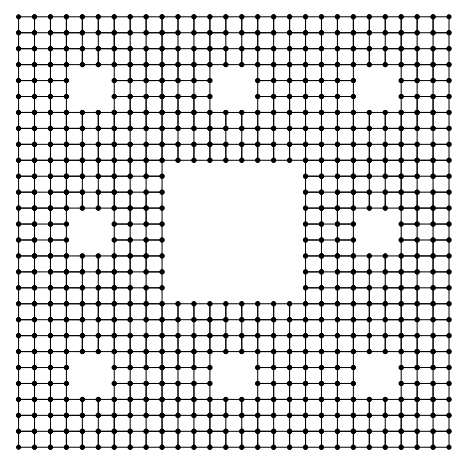

Fig. 1: The Sierpinski carpet graph.

denote with $z_{i}$ the connectivity of the site $i$, i.e. the number of its nearest neighbours.

Definition 2 A path in $X$ is a sequence of consecutive edges $\{(i, k)(k, h) \ldots(n, m)(m, j)\}$ and its length is the number of edges in the sequence. A graph is said to be connected if, for any two vertices $i, j \in V_{X}$, there is always a path joining them.

Definition 3 The adjacency matrix $A_{i j}$ is:

$$
A_{i j}= \begin{cases}1 & \text { if }(i, j) \in E_{X} \\ 0 & \text { if }(i, j) \notin E_{X}\end{cases}
$$

Unoriented graphs are naturally provided by an intrinsic distance, which in physics is called the chemical distance $r_{i, j}$.

Definition $4 r_{i, j}$ is the length of the shortest path connecting the vertices $i$ and $j$.

The chemical distance defi nes on the graph the balls of radius $r \in \mathbb{N}$ and center $o \in V_{X}$. In the physical literature these subgraphs are called the Van Hove spheres $S_{o, r}$.

Definition $5 \mathcal{S}_{o, r}$ is the subgraph of $X$, given by the set of vertices $V_{o, r}=\left\{i \in V \mid r_{i, o} \leq r\right\}$ and by the set of edges $E_{o, r}=\left\{(i, j) \in E \mid i \in V_{o, r}, j \in V_{o, r}\right\}$. We also define the border of $\mathcal{S}_{o, r}$ as the set of sites $\partial V_{o, r}=\left\{i \in V_{r}|\exists j \in X|,(i, j) \notin E_{o, r}\right\}$.

Let $|S|$ be the cardinality of a set $S$. Then $\left|V_{o, r}\right|$, as a function of the distance $r$, describes the growth rate of the graph at the large scales (MohWoe). In particular:

Definition 6 A graph is said to have a polynomial growth if $\forall o \in V_{X} \exists c, k$, such that $\left|V_{o, r}\right|<c r^{k}$ uniformly in $o$.

\section{The thermodynamic limit}

\subsection{Physical conditions}

Discrete structures describing real physical systems are characterized by some important properties, which can be translated in mathematical requirements. A graph $X$ will be called physical graph $\mathcal{G}$ if it satisfi es the following conditions and the sets of its vertices and edges will be denoted respectively with $V$ an $E$. 
p.c.1 We will consider only connected graphs (Defi nition 2), since any physical model on disconnected structures can be reduced to the separate study of the models defi ned on each connected component and hence to the case of connected graphs.

p.c.2 Since physical interactions are always bounded, the coordination numbers $z_{i}$, representing the number of neighbours interacting with the site $i$, have to be bounded; i.e. $\exists z_{\max } \mid z_{i} \leq z_{\max } \forall i \in V_{X}$. Notice that, in systems such as gasses and solids the Coulombian interaction is generally screened and it can be recast in terms of a short range coupling. Furthermore, when the long range interactions decrease rapidly enough, it can be shown (BC) that universal properties of the system behave as in the case of local couplings.

p.c.3 Real system are always embedded in 3-dimensional space. This constrain requires for the graph $\mathcal{G}$ the conditions:

(a) $X$ has a polynomial growth (Defi nition 6)

(b)

$$
\lim _{r \rightarrow \infty} \frac{\left|\partial V_{o, r}\right|}{\left|V_{o, r}\right|}=0
$$

The existence itself of the limit is a physical requirement on $\mathcal{G}$.

Some interesting graphs such as the Bethe lattices (Fig. 2) do not satisfy (a) and (b). For this kind of structures many results we give in this paper do not apply and one has to introduce different techniques.

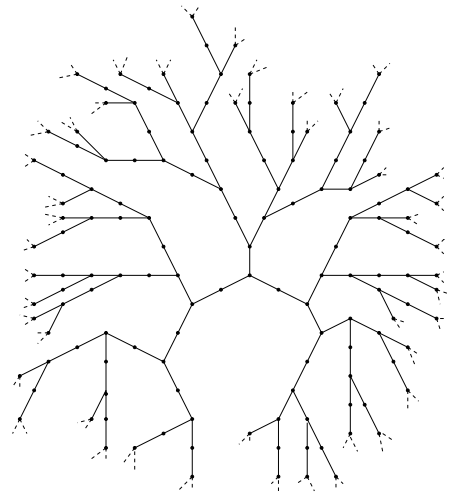

Fig. 2: An inhomogeneous Bethe lattice where $z_{i}$ has been randomly chosen equal to 2 or 3 .

\subsection{Averages in the thermodynamic limit and sets measure}

Thermodynamic averages have a crucial role in the study of statistical models on discrete structures. This requires the introduction on infi nite graphs of the Van Hove spheres and the thermodynamic limit. 
Definition 7 Given a physical graph $\mathcal{G}$, let $\phi: V \rightarrow \mathbb{R}$ The average in the thermodynamic limit of $\phi$ is:

$$
\bar{\phi} \equiv \lim _{r \rightarrow \infty} \frac{\sum_{i \in V_{o, r}} \phi_{i}}{\left|V_{o, r}\right|} .
$$

The existence itself of limit (3) is a physical requirement on $\phi$.

Remark The existence of the limit (3) is always assumed in the paper. Indeed functions for which (3) is not defi ned do not represent a well behaved thermodynamic quantity. From this point of view an interesting mathematical question regards the general conditions for the existence of the limit.

Physical constrains on graph structures, given in Section 2.1, have important consequences for the behaviour of the thermodynamic averages, such as the independence of the limit (3) from the choice of the center $o$.

Theorem 1 Let $\mathcal{G}$ be a physical graph and $\phi: V \rightarrow \mathbb{R}$ a function bounded from below, i.e. $\phi_{i}>\phi_{\min }>$ $-\infty \forall i \in V$. If limit (3) exists for the Van Hove spheres of center $o^{\prime}$, then it exists for any possible center $o$ and the result does not depend on 0 .

See e.g. (BCV1) for a proof.

In the following then we drop the index $o$ when we evaluate thermodynamic averages. Now we can defi ne the measure of the subsets of $V^{\prime} \subset V$.

Definition 8 Given a physical graph $\mathcal{G}$, let $V^{\prime} \subset V$ the measure of $V^{\prime}$ is $\left\|V^{\prime}\right\|=\overline{\chi\left(V^{\prime}\right)}$, where $\chi\left(V^{\prime}\right)$ is the characteristic function defined by $\chi_{i}\left(V^{\prime}\right)=1$ if $i \in V^{\prime}$ and $\chi_{i}\left(V^{\prime}\right)=0$ if $i \notin V^{\prime}$. The measure of a subset of edges $E^{\prime} \subset E$ is $\lim _{r \rightarrow \infty}\left|E_{r}^{\prime}\right| /\left|V_{r}\right|$, where $E_{r}^{\prime}=\left\{(i, j) \in E^{\prime} \mid i \in V_{r}, j \in V_{r}\right\}$.

Since $\chi\left(V^{\prime}\right)$ is bounded from below, when the thermodynamic average exists, the value of the measure $\left\|V^{\prime}\right\|$ does not depend on the choice of the center $o$. Unfortunately, in some cases the limit defi ning the measure does not exist. A typical example is the subsets of $\mathbb{Z}$ given by $\left\{i \in \mathbb{Z}\left|2^{2 n} \leq\right| i \mid \leq 2^{2 n+1}, \forall n \in \mathbb{N}\right\}$. However, these subsets are not very interesting from a physical point of view, for example they cannot characterize sites with a peculiar thermodynamic property, since this property should not be additive. Hence we will consider only subsets with a well defi ned measure.

\section{Random walks}

\subsection{The local type problem}

Let us begin by recalling the basic defi nitions and results concerning (simple) random walks on infi nite graphs. A more detailed treatment can be found in the mathematical reviews by Woess (W1; W2).

Definition 9 The (simple) random walk on a graph $X$ is defined by the jumping probability $p_{i j}$ between nearest neighbours sites $i$ and $j$ :

$$
p_{i j}=\frac{A_{i j}}{z_{i}}=\left(Z^{-1} A\right)_{i j}
$$

where $Z_{i j}=z_{i} \delta_{i j}$. The probability of reaching in $t$ steps site $j$ starting from $i$ is $P_{i j}(t)=\left(p^{t}\right)_{i j}$. We denote with $F_{i j}(t)$ the probability for a walker starting from $i$ of reaching for the first time in $t$ steps the site $j \neq i$ and with $F_{i i}(t)$ is the probability of returning to the starting point $i$ for the first time after $t$ steps $\left(F_{i i}(0)=0\right)$. 
The basic relationship between $P_{i j}(t)$ and $F_{i j}(t)$ is given by:

$$
P_{i j}(t)=\sum_{k=0}^{t} F_{i j}(k) P_{j j}(t-k)+\delta_{i j} \delta_{t 0}
$$

$F_{i j} \equiv \sum_{t=0}^{\infty} F_{i j}(t)$ turns out to be the probability of ever reaching the site $j$ starting from $i$ (or of ever returning to $i$ if $j=i$ ). Therefore $0<F_{i j} \leq 1$.

Definition 10 The generating functions $\tilde{P}_{i j}(\lambda)$ and $\tilde{F}_{i j}(\lambda)$ are given by:

$$
\tilde{P}_{i j}(\lambda)=\sum_{t=0}^{\infty} \lambda^{t} P_{i j}(t) \quad \tilde{F}_{i j}(\lambda)=\sum_{t=0}^{\infty} \lambda^{t} F_{i j}(t)
$$

where $\lambda$ is a complex number.

Multiplying equations (5) by $\lambda^{t}$ and then summing over all possible $t$ we get:

$$
\tilde{P}_{i j}(\lambda)=\tilde{F}_{i j}(\lambda) \tilde{P}_{j j}(\lambda)+\delta_{i j}
$$

In the following we will call $\tilde{P}_{i}(\lambda) \equiv \tilde{P}_{i i}(\lambda)$ and $\tilde{F}_{i}(\lambda) \equiv \tilde{F}_{i i}(\lambda)$.

Infi nite graphs can be classifi ed by the long time asymptotic behaviour of simple random walks and in particular by the quantities $\tilde{F}_{i}(1)$ and $\lim _{\lambda \rightarrow 1} \tilde{P}_{i}(\lambda)(\mathrm{P})$.

Definition 11 A graph $X$ is called locally recurrent if

$$
\tilde{F}_{i}(1)=1 \text { or equivalently } \lim _{\lambda \rightarrow 1} \tilde{P}_{i}(\lambda)=\infty \forall i \in V_{X}
$$

On the other hand, $X$ is called locally transient if:

$$
\tilde{F}_{i}(1)<1 \text { or equivalently } \lim _{\lambda \rightarrow 1} \tilde{P}_{i}(\lambda)<\infty \forall i \in V_{X}
$$

The equivalences in the defi nitions (8) and (9) are simple consequences of equation (7). By standard Markov chains properties one can prove (W1) that (8) and (9) are independent of the vertex $i$ and then Defi nition 11 can be considered as a property of the graph itself.

\subsection{The type problem on the average}

The study of thermodynamic properties of physical models requires the introduction of averages. In many many cases, such as spin models with $O(n)$ continuous symmetry, the thermodynamic behaviour is related to random walks by the return probabilities on the average (BCV1). In particular, physical arguments show that $O(n)$ spin systems present spontaneous magnetization if they are defi ned on a transient on the average graph $(\mathrm{Ca} ; \mathrm{BCV} 2)$.

Definition 12 Let $\mathcal{G}$ a physical graph such that the averages $\overline{F(\lambda)} \equiv \overline{\tilde{F}(\lambda)}$ and $\overline{P(\lambda)} \equiv \overline{\tilde{P}(\lambda)}$ are well defined. We say that $\mathcal{G}$ is recurrent on the average (ROA) if $\lim _{\lambda \rightarrow 1} \overline{F(\lambda)}=1$, while it is transient on the average (TOA) when $\lim _{\lambda \rightarrow 1} \overline{F(\lambda)}<1$. 
Remark The main mathematical point in defi nition 12 is the existence of the thermodynamic average for the functions $\tilde{F}_{i}(\lambda)$ and $\tilde{P}_{i}(\lambda)$. The existence of this limit will be always assumed on physical graphs. In (BZ) a case in which $\overline{P(\lambda)}$ and $\overline{F(\lambda)}$ are not well defi ned is presented. However this case is given by a graph $X$ not satisfying p.c.3. On the other hand $\overline{P(\lambda)}$ and $\overline{F(\lambda)}$ are well defi ned for all physical graph $\mathcal{G}$ we have studied up to now. A general result in this direction would be an important breakthrough to understand the average properties of random walks on graphs.

In the hypothesis of the existence of the thermodynamic averages, the limit $\lambda \rightarrow 1^{-}$is always well defi ned since $\overline{P(\lambda)}$ and $\overline{F(\lambda)}$ are increasing functions of $\lambda$. Furthermore the independence of the averages from the center of the spheres is assured by Theorem 1 and by the boundedness from below of $\tilde{F}_{i}(\lambda)$ and $\tilde{P}_{i}(\lambda)$. Hence Defi nition 12 represents a property of the graph.

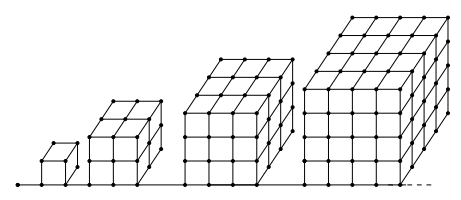

Fig. 3: Chain of increasing cubes.

In (BZ) a different defi nition for transience and recurrence on the average is given. There the thermodynamic limit $\lim _{r \rightarrow \infty}$ is replaced by the $\liminf _{r \rightarrow \infty}$ which is always well defi ned. Moreover in (BZ) the limit $\lambda \rightarrow 1^{-}$is evaluated before the thermodynamic average. This defi nition leads to a new graph classifi cation. For example the chain of increasing cubes (see Fig. 3) from Defi nition 12 is a TOA graph, while following the defi nition of (BZ) it is recurrent on the average. Furthermore, the condition for the independence of the limit from the center of the sphere, in this case is weaker than the hypotheses of Section 2.1.

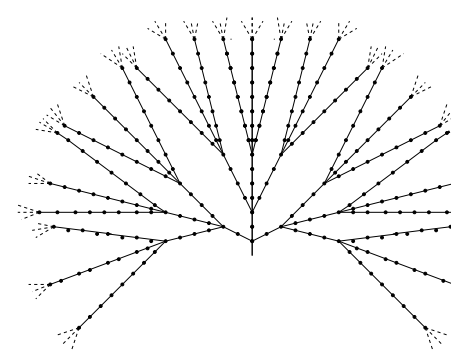

Fig. 4: The NTD graph.

Recurrence and transience on the average are in general independent from the corresponding local properties. The fi rst example of this phenomenon occurring on inhomogeneous structures was found in a class of infi nite trees called NTD (Fig. 4) which are locally transient but recurrent on the average (BCR). On the other hand the chain of increasing cubes in Fig. 3 is an example of locally recurrent but transient on the averages graph. 
We cannot prove any simple relation between $\overline{F(\lambda)}$ and $\overline{P(\lambda)}$ analogous to equation (7) for local probabilities. Indeed averaging (7) over all sites $i$ would involve the average of a product, which, due to correlations, is in general different from the product of the average. Therefore, the double implication $\tilde{F}_{i}(1)=1 \Leftrightarrow \lim _{\lambda \rightarrow 1} \tilde{P}_{i}(\lambda)=\infty$ is not true. However, one can prove a weaker result.

Theorem 2 Let $\mathcal{G}$ a ROA graph (i.e. $\lim _{\lambda \rightarrow 1} \overline{F(\lambda)}=1$ ), then $\lim _{\lambda \rightarrow 1} \overline{P(\lambda)}=\infty$.

See (BCV1) for the proof.

On the other hand there are graphs for which $\lim _{\lambda \rightarrow 1} \overline{F(\lambda)}<1$ but $\lim _{\lambda \rightarrow 1} \overline{P(\lambda)}=\infty$ (an example is shown in Fig. 5).

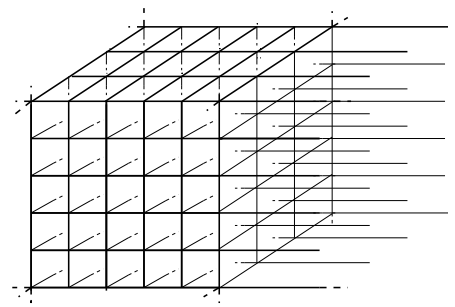

Fig. 5: The graph obtained as a union of a 3-dimensional lattice and linear chains.

\section{Percolation}

\subsection{Definitions}

On lattice, some important classical works (FK; ACCN) relate percolation with the properties of statistical physics models with discrete symmetry (typically Ising and Potts). On generic graphs one can extend these results showing that the thermodynamic properties of the system depend on the problem of percolation on the average (V). Let us fi rst defi ne percolation, see e.g. (F).

Definition 13 On a graph $X$, percolation is defined by declaring each link of the graph open with probability $p$ and closed with probability $1-p, 0 \leq p \leq 1$. We will call cluster $C_{i}$ the set of all sites connected to $i$ by a path of open links. The $i$-length $l$ of the cluster $C_{i}$ is the maximum chemical distance from $i$ of the sites in $C_{i}: l=\sup _{j \in C_{i}} r_{i, j}$ and $P_{i}(l, p)$ is the probability for the site $i$ to belong to a cluster of $i$-length $>l$.

The local percolation is defi ned as:

Definition 14 A graph presents (local) percolation if it exists a probability $p<1$ such that

$$
\lim _{l \rightarrow \infty} P_{i}(l, p)>0 .
$$

It can be shown the property does not depend on $i(F)$.

The study of thermodynamic properties of Ising and Potts model is related to a different classifi cation defi ned by percolation on the average. 
Definition 15 Let $\mathcal{G}$ a physical graph such that the average $\overline{P(l, p)}$ is well defined $\forall p, l$. $\mathcal{G}$ present percolation on the average if it exists a probability $p<1$ such that

$$
\lim _{l \rightarrow \infty} \overline{P(l, p)}>0 .
$$

Remark Also for (11) the main mathematical point regards the general conditions for the existence of the thermodynamic limit.

In (11) we consider an average of a bounded function, therefore defi nition 15 does not depend on the center of the sphere and it represents a characteristic of the graph. Furthermore $\overline{P(l, p)}$ is a decreasing function of $l$, then, if the average is well defi ned, the limit $l \rightarrow \infty$ always exists.

Average and local percolation are in general different properties which give rise to different classifi cations of physical graphs, an example is given by the brush graph in Fig. 6 which presents local percolation but no percolation on the average $(\mathrm{V})$.

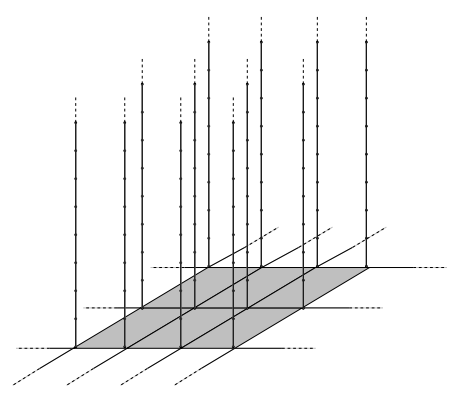

Fig. 6: The brush graph.

\subsection{Thermodynamic properties of the Potts model on graph}

Here we introduce the Potts model (see $(\mathrm{Wu})$ for a review) and illustrate the theorem which proves that the model exhibits spontaneous magnetization if and only if it is defi ned on a graph presenting percolation on the average.

Let us fi rst introduce the Potts model restricted to the sphere $\mathcal{S}$.

Definition 16 For each site $i \in V_{r}$ be $s_{i}$ a $q$ states function $s_{i}=1 \ldots q$. The Hamiltonian $H_{r}$ of the $q$-states Potts model on $\mathcal{S}_{r}$ is:

$$
H_{r}=\sum_{(i, j) \in E_{r}}\left(1-\delta\left(s_{i}, s_{j}\right)\right)+h \sum_{i \in V_{r}}\left(1-\delta\left(s_{i}, 1\right)\right)
$$

where the Kronecker delta-function is $\delta\left(s_{i}, s_{j}\right)=1$ if $s_{i}=s_{j}$ and $\delta\left(s_{i}, s_{j}\right)=0$ otherwise. The partition function $Z_{r}$ is given by the sum of the Boltzmann weight $\exp \left(-\beta H_{r}\right)$ over all the possible configuration $\left\{s_{i}\right\} ; \beta$ represents the inverse temperature of the system.

The case $q=2$ can be simply reduced to Ising model introducing the new variables $\sigma_{i}=2 s_{i}-3$. 
Thermodynamic properties of statistical models are described by extensive order parameters, in this case the average magnetization:

$$
M_{r}(\beta, q, h)=\left\langle\left|V_{r}\right|^{-1} \sum_{i \in V_{r}} \delta\left(s_{i}, 1\right)-q^{-1}\right\rangle_{r} \equiv Z_{r}^{-1} \sum_{\left\{s_{1} \ldots s_{V_{r}}\right\}}\left|V_{r}\right|^{-1} \sum_{i \in V_{r}}\left(\delta\left(s_{i}, 1\right)-q^{-1}\right) e^{-\beta H_{r}}
$$

where the thermal average $\langle\cdot\rangle_{r}$ is obtained by summing over all the possible confi gurations $\left\{s_{1} \ldots s_{S_{r}}\right\}$ weighted by $Z_{r}^{-1} \exp \left(-\beta H_{r}\right)$.

Definition 17 Let $\mathcal{G}$ a physical graph such that for all values of $\beta$, h and $q$ it exists the limit $\lim _{r \rightarrow \infty} M_{r}(\beta, q, h)$. The q-states Potts model presents spontaneous magnetization on $\mathcal{G}$ if it exists a temperature $\beta_{c}$ such that for all $\beta>\beta_{c}$

$$
\lim _{h \rightarrow 0} M(\beta, q, h) \equiv \lim _{h \rightarrow 0} \lim _{r \rightarrow \infty} M_{r}(\beta, q, h)>0 .
$$

Let us prove the theorem showing that the Potts model is spontaneously magnetized if and only if it is defi ned on a graph presenting percolation on the average. The result is inspired by a mathematical paper (Hä) where it is proven an analogous result relating (local) percolation and the existence of a single Gibbs measure for Potts model. The multiplicity of Gibbs measure well describes phase transitions from a probabilistic point of view, however it does not take into account of the thermodynamic properties of the system which are described by extensive parameters such as the average magnetization (13). We fi rst introduce a defi nition and two lemmas (a detailed version of the proof can be found in $(\mathrm{V})$ ).

Definition 18 For a given Van Hove sphere $\mathcal{S}_{r}$ we call supplemented sphere $\mathcal{S}_{r}^{\prime}$ the graph defined by the set of vertices $V_{r}^{\prime}=V_{r} \cup o$ and the set of links $E_{r}^{\prime}=E_{r} \cup\left\{(i, o), \forall i \in V_{r}\right\}$, where $o \notin V$ is a supplemented site. Let us consider the percolation on $S_{r}^{\prime}$. We declare each link $(i, j) \in V_{r}$ open with probability $p$ (closed with probability $1-p)$ and each link $(i, o)$ open with probability $p_{o}^{\prime}$ (closed with probability $\left.1-p_{o}^{\prime}\right)$. We will call $P_{i}^{o}\left(p, p_{o}\right)$ the probability for the site $i$ to belong to the same cluster of the supplemented site $o$.

Lemma 1 For the local magnetization $\left\langle\delta\left(s_{i}, 1\right)-q^{-1}\right\rangle_{r}$ the following upper and lower bounds hold

$$
P_{i}^{o}\left(p^{\prime}, p_{o}^{\prime}\right) \leq\left\langle\delta\left(s_{i}, 1\right)-q^{-1}\right\rangle_{r} \leq P_{i}^{o}\left(p, p_{o}\right)
$$

with $p=1-e^{-\beta}, p_{o}=1-e^{-\beta h}, p^{\prime}=p(q-p(q-1))^{-1}$ and $p_{o}^{\prime}=p_{o}\left(q-p_{o}(q-1)\right)^{-1}$.

The proof can be obtained from the random-cluster representation of the Potts model (FK) using statistical domination and FKG inequality (FKG) as it is shown in (Hä).

Lemma 2 Let us consider percolation on the sphere $S_{r}$ and call $P_{i, r}(l, p)$ the probability for the site $i$ to belong to a cluster of $i$-length larger than $l$. In the thermodynamic limits one can replace $P_{i, r}(l, p)$ with the probability defined on the infinite graph $P_{i}(l, p)$.

This property is a consequence of the physical conditions p.c.2, p.c.3 and the fact that, for sites $i$ at a distance from the border of the sphere greater than $l, P_{i, r}(l, p)=P_{i}(l, p)$.

Theorem 3 Let $\mathcal{G}$ a physical graph such that the thermodynamic limits in (11) and (14) are well defined. The Potts model on $\mathcal{G}$ is spontaneously magnetized if and only if the graph $\mathcal{G}$ exhibits percolation on the average. 
Let us consider $P_{i}^{o}\left(p, p_{o}\right)$ from p.c.2 and the independence of percolation probabilities, one gets:

$$
P_{i}^{o}\left(p, p_{o}\right) \leq\left(1-\left(1-p_{o}\right)^{z_{\max }^{l}}\right)\left(1-P_{i, r}(l, p)\right)+P_{i, r}(l, p) .
$$

Indeed $\left(z_{\max }^{l}-1\right) /\left(z_{\max }+1\right)$ is the maximum number of sites in a cluster of i-length $<l$ and then $(1-$ $\left.\left(1-p_{o}\right)^{z_{\max }^{l}}\right)$ is an upper bound on the probability that a site of the cluster is connected to $o$. Taking the thermodynamic average in in (16), from lemmas 1 and 2 one obtains:

$$
\lim _{h \rightarrow 0} M(\beta, q, h) \leq \lim _{h \rightarrow 0}\left(1-\left(1-p_{o}\right)^{z_{\max }^{l}}\right)(1-\overline{P(l, p)})+\overline{P(l, p)}=\overline{P(l, p)} .
$$

Furthermore we have

$$
P_{i}^{o}\left(p^{\prime}, p_{o}^{\prime}\right) \geq\left(1-\left(1-p_{o}^{\prime}\right)^{l+1}\right) P_{i, r}\left(l, p^{\prime}\right) .
$$

Here $l+1$ is the minimum number of sites in a cluster of $i$-length $\geq l$, and then $\left(1-\left(1-p_{o}^{\prime}\right)^{l+1}\right)$ is a lower bound on the probability that a site of the cluster is connected to $o$. Taking the thermodynamic average and letting $l \rightarrow \infty$ we have

$$
M(\beta, q, h)=\lim _{l \rightarrow \infty} M(\beta, q, h) \geq \lim _{l \rightarrow \infty}\left(1-\left(1-p_{o}^{\prime}\right)^{l+1}\right) \overline{P\left(l, p^{\prime}\right)}=\lim _{l \rightarrow \infty} \overline{P\left(l, p^{\prime}\right)}
$$

Letting $l \rightarrow \infty$ in (17) and $h \rightarrow 0$ in (19) one has

$$
\lim _{l \rightarrow \infty} \overline{P\left(l, p^{\prime}\right)} \leq \lim _{h \rightarrow 0} M(\beta, q, h) \leq \lim _{l \rightarrow \infty} \overline{P(l, p)}
$$

If the graph presents percolation on the average, it exists a probability $p^{\prime}$ such that $\lim _{l \rightarrow \infty} \overline{P\left(l, p^{\prime}\right)}>0$ and then the fi rst inequality in (20) implies that the model for $\beta=\log \left(1+p^{\prime} q\left(1-p^{\prime}\right)^{-1}\right)$ is magnetized. On the other hand, if $\lim _{l \rightarrow \infty} \overline{P(l, p)}=0$ for all values of $p$, the second inequality in (20) implies that the magnetization of the system is zero for all temperatures and this concludes the proof.

\section{References}

[ACCN] Aizenman M, Chayes JT, Chaies L and Newman CM 1988 Jour. Stat. Phys. 501

[AO] Alexander S and Orbach R 1987 J. Phys. (Paris) Lett. 92108

[B] Biggs NL 1974 Algebraic graph theory Cambridge University Press

[BBC] Buonsante P, Burioni R, Cassi D, Meccoli I, Regina S and Vezzani A 2000 Physica A 280131

[BC] Burioni R and Cassi D 1997 Mod. Phys. Lett. B 111095

[BCR] Burioni R, Cassi D and Regina S 1996 Mod. Phys. Lett. B 101059

[BCV1] Burioni R, Cassi D and Vezzani A 2000 Eur. Phys. J.B. 15665

[BCV2] Burioni R, Cassi D and Vezzani A 1999 Jour. Phys. A 325539

[BZ] Bertacchi D and Zucca F, preprint arxiv.org mathPR/0104252. 
[Ca] Cassi D 1996 Phys. Rev. Lett. 762941

[CF] D. Cassi and L. Fabbian 1999 Jour. Phys. A 32 L93

[CDS] Cvetković DM, Doob M and Sachs H 1979 Spectra of graphs Deutsccher Verlag der Wissenschaften, Berlin and Accademic Press new York

[F] Fortuin CM 1972 Physica 58393

[FK] Fortuin CM and Ksteleyn PW 1972 Physica 57536

[FKG] Fortuin CM, Ksteleyn PW and Ginibre J 1972 Commun. Math. Phys 22 89;

[FSS] Fröhlich J, Simon B and Spencer T 1976 Commun. Math. Phys. 5079

[H] Harari F 1969 Graph theory Addison-Wesley Reading

[Hä] Häggström O 2000 Adv. Appl. Prob. 39

[HHW] Hattori K, Hattori T and Watanabe H 1987 Prog. Theor. Phys. Suppl. 92108

[MohWoe] Mohar B and Woess W 1989 Bull. London Math. Soc. 21209

[MW] Mermin ND and Wagner H 1966 Phys. Rev. 171133

[P] Polya G 1921Math. Ann. 84149

[PG] Griffi ths RB 1964 Phys. Rev. 136437

[V] Vezzani A preprint arxiv.org cond-mat/0306167

[S] Shinoda M 2002 J. App. Prob. 391

[W1] Woess W 1994 Bull. London Math. Soc. 261

[W2] Woess W 2000 Random walks on infinite graphs and groups, Cambridge University Press 2000

[Wu] Wu FY 1982 Rev. Mod. Phys. 54235 